Article

\title{
Anti-Intuitionistic Fuzzy Soft a-Ideals Applied to BCI-Algebras
}

\author{
G. Muhiuddin $1, * \mathbb{( D}$, D. Al-Kadi ${ }^{2}$ and M. Balamurugan ${ }^{3}$
}

1 Department of Mathematics, University of Tabuk, Tabuk 71491, Saudi Arabia

2 Department of Mathematics and Statistics, Taif University, Taif 21974, Saudi Arabia; dak12le@hotmail.co.uk

3 Department of Mathematics, Sri Vidya Mandir Arts and Science College (Autonomous),

Uthangarai 636902, Tamilnadu, India; balamurugansvm@gmail.com

* Correspondence: chishtygm@gmail.com

Received: 2 June 2020; Accepted: 29 June 2020; Published: 8 July 2020

\begin{abstract}
The notion of anti-intuitionistic fuzzy soft a-ideals of $B C I$-algebras is introduced and several related properties are investigated. Furthermore, the operations, namely; AND, extended intersection, restricted intersection, and union on anti-intuitionistic fuzzy soft a-ideals are discussed. Finally, characterizations of anti-intuitionistic fuzzy soft a-ideals of $B C I$-algebras are given.
\end{abstract}

Keywords: $B C I$-algebras; soft set; fuzzy soft set; intuitionistic fuzzy soft set; anti-intuitionistic fuzzy soft ideals; anti-intuitionistic fuzzy soft a-ideals

MSC: 06F35; 03G25; 06D72

\section{Introduction}

The theory of fuzzy set, intuitionistic fuzzy sets, soft set, and more other theories were introduced to deal with uncertainty. In [1], Zadeh introduced the concept of a fuzzy subset of a set. Later on, a number of generalizations of this fundamental notion have been studied by many authors in different directions. The notion of an intuitionistic fuzzy set defined in [2] is a generalization of a fuzzy set. It gives more opportunity to be accurate when dealing with uncertain objects. Soft set theory was initially suggested by Molodstov in [3], then Maji et al. in [4] combined the soft set theory and the intuitionistic fuzzy set theory, and introduced the notion intuitionistic fuzzy soft sets.

Algebra is the language in which combinatorics are usually expressed. Combinatorics is the study of discrete structures that arise not only in areas of pure mathematics, but in other areas of science, for example, computer science, statistical physics and genetics. From ancient beginnings, this subject truly rose to prominence from the mid-20th century, when scientific discoveries (most notably of DNA) showed that combinatorics is key to understanding the world around us, whilst many of the great advances in computing were built on combinatorial foundations. These concepts were widely studied over different classes of logical algebras as the essential classes of $B C K / B C I$-algebras presented by Iseki [5]. The concepts intuitionistic fuzzy ideals of $B C K$-algebras were studied in [6]. Bej et al. [7] declared the concept of doubt intuitionistic fuzzy subalgebra and doubt intuitionistic fuzzy ideal in $B C K / B C I$-algebras. Muhiuddin et al. studied various concepts on fuzzy sets and applied them to $B C K / B C I$-algebras, and other related notions (see for e.g., [8-18]). Also, some new generalizations of fuzzy sets and other related concepts in different algebras have been studied in (see for e.g., [6,19-35]). Additionally, Balamurugan et al. [36] introduced the concepts of intuitionistic fuzzy soft subalgebras, intuitionistic fuzzy soft ideals, and intuitionistic fuzzy soft a-ideals of $B$-algebra and studied several properties of these notions. 
In the present paper, we introduce the notion of anti-intuitionistic fuzzy soft a-ideals in BCI-algebras. The results of present paper are organized, as follows: Section 2 summarizes some basic definitions and properties that are needed to develop our main results while in Section 3, we introduce the notion of anti-intuitionistic fuzzy soft a-ideals of $B C I$-algebras and investigate related properties. In Section 4, we give characterizations of anti-intuitionistic fuzzy soft a-ideals of $B C I$-algebras while using the concept of a soft level set.

\section{Preliminaries}

In this section, we recall basic definitions and results that are related to the subject of the paper.

Definition 1. [5] An algebra $(\Omega ; \odot, 0)$ of type $(2,0)$ is called a BCI-algebra if it satisfies the following conditions:

(1) $\quad((l \odot m) \odot(l \odot n)) \odot(n \odot m)=0$,

(2) $(l \odot(l \odot m)) \odot m=0$,

(3) $l \odot l=0$;

(4) $l \odot m=0$ and $m \odot l=0 \Rightarrow l=m$, for all $l, m, n \in \Omega$.

Any $B C I$-algebra $\Omega$, satisfies the following axioms:

(I) $l \odot 0=l$,

(II) $l \leq m \Rightarrow l \odot n \leq m \odot n$ and $n \odot m \leq n \odot l$,

(III) $(l \odot n) \odot(m \odot n) \leq l \odot m$,

(IV) $0 \odot(0 \odot(l \odot m))=(0 \odot m) \odot(0 \odot l)$,

(V) $(l \odot m) \odot n=(l \odot n) \odot m$,

where $l \leq m \Leftrightarrow l \odot m=0$, for any $l, m, n \in \Omega$.

A non-empty subset $\Delta$ of a $B C K$-algebra $\Omega$ is called an ideal of $\Omega$ if it satisfies

(1) $0 \in \Delta$,

(1) $\forall l, m \in \Omega, l * m \in \Delta, m \in \Delta \Rightarrow l \in \Delta$.

A non-empty subset $\Delta$ of a $B C K$-algebra $\Omega$ is called an a-ideal of $\Omega$ if it satisfies (1) and (3) $\forall l, m \in \Omega,(l \odot n) \odot(0 \odot m) \in \Delta, n \in \Delta \Rightarrow m \odot l \in \Delta$.

For an initial set $\Omega$ and a set of parameters $\Delta$, a pair $(Y, \Delta)$ is said to be a soft set over $\Omega \Leftrightarrow \exists Y$ : $\Delta \rightarrow \wp(\Omega)$, where $\wp(\Omega)$ is a family of subsets of $\Omega$. (see [30] for more details on soft set theory).

Definition 2. [4] Let $\Pi$ be a collection of parameters and let $Y(\Omega)$ indicate the collection of all fuzzy sets in $\Omega$. Then $(\mathrm{Y}, \Delta)$ is called a fuzzy soft set over $\Omega$, where $\Delta \subseteq \Pi$ and $\mathrm{Y}: \Delta \rightarrow \mathrm{Y}(\Omega)$.

Definition 3. [36] Let $(Y, \Delta)$ be a fuzzy soft set (abbr. FSS). Then $(Y, \Delta)$ is an anti-fuzzy soft ideal (abbr. AFSID) of $\Omega$ if $\mathrm{Y}[\boldsymbol{\omega}]=\left\{\left(\xi_{\mathrm{Y}[\omega]}(l)\right): l \in \Omega\right.$ and $\left.\mathfrak{\omega} \in \Delta\right\}$ is an AFID of $\Omega$ satisfies the following assertions:

(i) $\xi_{Y[\omega]}(0) \leq \xi_{Y[\omega]}(l)$,

(ii) $\xi_{Y[\omega]}(l) \leq \xi_{Y[\omega]}(l \odot m) \vee \xi_{Y[\omega]}(m)$,

for all $l, m, n \in \Omega$ and $\omega \in \Delta$.

Definition 4. [36] Let $(\mathrm{Y}, \Delta)$ be a fuzzy soft set (abbr. FSS). Then $(\mathrm{Y}, \Delta)$ is an anti-fuzzy soft a-ideal (abbr. AFSID) of $\Omega$ if $\mathrm{Y}[\omega]=\left\{\left(\xi_{\mathrm{Y}[\omega]}(l)\right): l \in \Omega\right.$ and $\left.\omega \in \Delta\right\}$ is an AFID of $\Omega$ satisfies the following assertions:

(i) $\xi_{Y[\omega]}(0) \leq \xi_{Y[\omega]}(l)$, 
(ii) $\quad \xi_{Y[\omega]}(m \odot l) \leq \xi_{Y[\omega]}((l \odot n) \odot(0 \odot m)) \vee \xi_{Y[\omega]}(n)$,

for all $l, m, n \in \Omega$ and $\omega \in \Delta$.

Definition 5. [4] Let $\Pi$ be a collection of parameters and let $\mathbb{I} Y(\Omega)$ indicate the collection of all intuitionistic fuzzy sets in $\Omega$. Subsequently, $(\mathrm{Y}, \Delta)$ is called an intuitionistic fuzzy soft set over $\Omega$, where $\Delta \subseteq \Pi$ and $\mathrm{Y}: \Delta \rightarrow \mathbb{I Y}(\Omega)$.

\section{Anti-Intuitionistic Fuzzy Soft a-Ideal}

In what follows, we write $\Omega$ to denote a $B C I$-algebra $(\Omega ; \odot, 0)$ and $\operatorname{IFS}$ for intuitionistic fuzzy sets and we will introduce an abbreviation for the notions in the following definitions to be used in the rest of the paper.

Definition 6. Let $(\mathrm{Y}, \Delta)$ be an intuitionistic fuzzy soft set (abbr. IFSS). Afterwards, $(\mathrm{Y}, \Delta)$ is an anti-intuitionistic fuzzy soft ideal (abbr. AIFSID) of $\Omega$ if $\mathrm{Y}[\omega]=\left\{\left(\xi_{\mathrm{Y}[\omega]}(l), \zeta_{\mathrm{Y}[\omega]}(l)\right): l \in \Omega\right.$ and $\omega \in \Delta\}$ is an AIFID of $\Omega$ satisfies the following assertions:

(i) $\xi_{\mathrm{Y}[\omega]}(0) \leq \xi_{\mathrm{Y}[\omega]}(l)$ and $\zeta_{\mathrm{Y}[\omega]}(0) \geq \zeta_{\mathrm{Y}[\omega]}(l)$,

(ii) $\xi_{Y[\omega]}(l) \leq \xi_{Y[\omega]}(l \odot m) \vee \xi_{Y[\omega]}(m)$,

(iii) $\zeta_{\mathrm{Y}[\omega]}(l) \geq \zeta_{\mathrm{Y}[\omega]}(l \odot m) \wedge \zeta_{\mathrm{Y}[\omega]}(m)$,

for all $l, m, n \in \Omega$ and $\omega \in \Delta$.

Definition 7. An IFSS $(\mathrm{Y}, \Delta)$ is called an anti-intuitionistic fuzzy soft a-ideal (abbr. AIFSAID) of $\Omega$ if $\mathrm{Y}[\omega]=\left\{\left(\xi_{\mathrm{Y}[\omega]}(l), \zeta_{\mathrm{Y}[\omega]}(l)\right): l \in \Omega\right.$ and $\left.\omega \in \Delta\right\}$ is an AIFAID of $\Omega$ satisfies the following assertions:

(i) $\quad \xi_{\mathrm{Y}[\omega]}(0) \leq \xi_{\mathrm{Y}[\omega]}(l)$ and $\zeta_{\mathrm{Y}[\omega]}(0) \geq \zeta_{\mathrm{Y}[\omega]}(l)$,

(ii) $\quad \xi_{Y[\omega]}(m \odot l) \leq \xi_{Y[\omega]}((l \odot n) \odot(0 \odot m)) \vee \xi_{Y[\omega]}(n)$,

(iii) $\zeta_{\mathrm{Y}[\omega]}(m \odot l) \geq \zeta_{\mathrm{Y}[\omega]}((l \odot n) \odot(0 \odot m)) \wedge \zeta_{\mathrm{Y}[\omega]}(n)$,

for all $l, m, n \in \Omega$ and $\omega \in \Delta$.

Example 1. Suppose that there are four patients in the initial universe set $\Omega=\left\{p_{1}, p_{2}, p_{3}, p_{4}\right\}$ given by

\begin{tabular}{|c|c|c|c|c|}
\hline$\odot$ & $p_{1}$ & $p_{2}$ & $p_{3}$ & $p_{4}$ \\
\hline$p_{1}$ & $p_{1}$ & $p_{2}$ & $p_{3}$ & $p_{4}$ \\
\hline$p_{2}$ & $p_{2}$ & $p_{1}$ & $p_{4}$ & $p_{3}$ \\
\hline$p_{3}$ & $p_{3}$ & $p_{4}$ & $p_{1}$ & $p_{2}$ \\
\hline$p_{4}$ & $p_{4}$ & $p_{3}$ & $p_{2}$ & $p_{1}$ \\
\hline
\end{tabular}

Afterwards, $\left(\Omega ; \odot, p_{1}\right)$ is a BCI-algebra.

Let a set of parameters, we consider $\Delta=\{f, s, n\}$ be a status of patients, in which

$f$ stands for the parameter "fever" can be treated by antibiotic,

$s$ stands for the parameter "sneezing" can be treated by antiallergic,

$n$ stands for the parameter "nosal block" can be treated by nosal drops.

Subsequently, $\mathrm{Y}[f], \mathrm{Y}[s]$, and $\mathrm{Y}[n]$ are IFSs over $\Omega$ represented by:

\begin{tabular}{|c|c|c|c|c|}
\hline $\mathrm{Y}$ & $p_{1}$ & $p_{2}$ & $p_{3}$ & $p_{4}$ \\
\hline$f$ & {$[0.1,0.8]$} & {$[0.1,0.8]$} & {$[0.2,0.6]$} & {$[0.2,0.6]$} \\
\hline$s$ & {$[0.0,0.9]$} & {$[0.0,0.9]$} & {$[0.3,0.7]$} & {$[0.3,0.7]$} \\
\hline$n$ & {$[0.2,0.7]$} & {$[0.2,0.7]$} & {$[0.4,0.6]$} & {$[0.4,0.6]$} \\
\hline
\end{tabular}

Therefore, $\mathrm{Y}[f], \mathrm{Y}[\mathrm{s}]$, and $\mathrm{Y}[n]$ are an $A I F A I D$ of $\Omega$ with respect to $f, s$, and $n$, respectively.

Hence, $(\mathrm{Y}, \Delta)$ is an AIFSAID of $\Omega$. 
Proposition 1. For any AIFSAID $(\mathrm{Y}, \Delta)$ of $\Omega$, the following inequalities hold:

$\xi_{Y[\omega]}(m \odot l) \leq \xi_{Y[\omega]}(l \odot(0 \odot m))$ and $\zeta_{Y[\omega]}(m \odot l) \geq \zeta_{Y[\omega]}(l \odot(0 \odot m))$, for any $\omega \in \Delta$ and $l, m \in \Omega$.

Proof. Let $(\mathrm{Y}, \Delta)$ be an AIFSAID of $\Omega$.

Subsequently, $\mathrm{Y}[\omega]=\left\{\left(\xi_{\mathrm{Y}[\omega]}(l), \zeta_{\mathrm{Y}[\omega]}(l)\right): l \in \Omega\right.$ and $\left.\omega \in \Delta\right\}$ is an AIFAID of $\Omega$.

Thus, for every $l, m, n \in \Omega$ and $\omega \in \Delta$,

and

$$
\xi_{Y[\omega]}(m \odot l) \leq \xi_{Y[\omega]}((l \odot n) \odot(0 \odot m)) \vee \xi_{Y[\omega]}(n)
$$

$$
\zeta_{Y[\omega]}(m \odot l) \geq \zeta_{Y[\omega]}((l \odot n) \odot(0 \odot m)) \wedge \zeta_{Y[\omega]}(n) .
$$

By substituting $n=0$, we get,

$$
\begin{gathered}
\xi_{Y[\omega]}(m \odot l) \leq \xi_{Y[\omega]}((l \odot 0) \odot(0 \odot m)) \vee \xi_{Y[\omega]}(0) \\
=\xi_{Y[\omega]}(l \odot(0 \odot m)) \vee \xi_{Y[\omega]}(0) \\
\zeta_{Y[\omega]}(m \odot l) \leq \xi_{Y[\omega]}(l \odot(0 \odot m)) \\
\zeta_{Y[\omega]}(m \odot l) \geq \zeta_{Y[\omega]}((l \odot 0) \odot(0 \odot m)) \wedge \zeta_{Y[\omega]}(0) \\
=\zeta_{Y[\omega]}(l \odot(0 \odot m)) \wedge \zeta_{Y[\omega]}(0) \\
\zeta_{Y[\omega]}(m \odot l) \geq \zeta_{Y[\omega]}(l \odot(0 \odot m)) .
\end{gathered}
$$

and

Theorem 1. Over $\Omega$, any AIFSAID is an AIFSID.

Proof. Let $(\mathrm{Y}, \Delta)$ be an AIFSAID of $\Omega$.

Subsequently, $\mathrm{Y}[\omega]=\left\{\left(\xi_{\mathrm{Y}[\omega]}(l), \zeta_{\mathrm{Y}[\omega]}(l)\right): l \in \Omega\right.$ and $\left.\omega \in \Delta\right\}$ is an AIFAID of $\Omega$.

Thus, for every $l, m, n \in \Omega$ and $\omega \in \Delta$,

and

$$
\xi_{Y[\omega]}(m \odot l) \leq \xi_{Y[\omega]}((l \odot n) \odot(0 \odot m)) \vee \xi_{Y[\omega]}(n)
$$

$$
\zeta_{Y[\omega]}(m \odot l) \geq \zeta_{Y[\omega]}((l \odot n) \odot(0 \odot m)) \wedge \zeta_{Y[\omega]}(n) .
$$

By substituting $l=0$ we obtain,

and

$$
\xi_{Y[\omega]}(m \odot 0) \leq \xi_{Y[\omega]}((0 \odot n) \odot(0 \odot m)) \vee \xi_{Y[\omega]}(n)
$$

$$
\zeta_{Y[\omega]}(m \odot 0) \geq \zeta_{Y[\omega]}((0 \odot n) \odot(0 \odot m)) \wedge \zeta_{Y[\omega]}(n) .
$$

Because we know that $(0 \odot n) \odot(0 \odot m) \leq m \odot n$, therefore

and

$$
\xi_{Y[\omega]}((0 \odot n) \odot(0 \odot m)) \leq \xi_{Y[\omega]}(m \odot n)
$$

Thus,

$$
\zeta_{Y[\omega]}((0 \odot n) \odot(0 \odot m)) \geq \zeta_{Y[\omega]}(m \odot n) .
$$

and

$$
\xi_{Y[\omega]}(m) \leq \xi_{Y[\omega]}((0 \odot n) \odot(0 \odot m)) \vee \xi_{Y[\omega]}(n) \leq \xi_{Y[\omega]}(m \odot n) \vee \xi_{Y[\omega]}(n)
$$

$\zeta_{Y[\omega]}(m) \geq \zeta_{Y[\omega]}((0 \odot n) \odot(0 \odot m)) \wedge \zeta_{Y[\omega]}(n) \geq \zeta_{Y[\omega]}(m \odot n) \wedge \zeta_{Y[\omega]}(n)$,

i.e., $\mathrm{Y}[\omega]=\left\{\left(\xi_{\mathrm{Y}[\omega]}(l), \zeta_{\mathrm{Y}[\omega]}(l)\right): l \in \Omega\right.$ and $\left.\omega \in \Delta\right\}$ is an AIFID of $\Omega$.

Hence $(\mathrm{Y}, \Delta)$ is an AIFSID of $\Omega$.

The converse of Theorem 1 is not true in general i.e., an AIFSID might not be an AIFSAID, as shown in the next example and we will give in the latter theorem a condition for this converse to be true.

Example 2. Let $\Omega=\{0, p, q, r, s\}$ with Cayley table: 


\begin{tabular}{|c|c|c|c|c|c|}
\hline$\odot$ & 0 & $\mathrm{p}$ & $\mathrm{q}$ & $\mathrm{r}$ & $\mathrm{s}$ \\
\hline 0 & 0 & 0 & $\mathrm{~s}$ & $\mathrm{r}$ & $\mathrm{q}$ \\
\hline $\mathrm{p}$ & $\mathrm{p}$ & 0 & $\mathrm{~s}$ & $\mathrm{r}$ & $\mathrm{q}$ \\
\hline $\mathrm{q}$ & $\mathrm{q}$ & $\mathrm{q}$ & 0 & $\mathrm{~s}$ & $\mathrm{r}$ \\
\hline $\mathrm{r}$ & $\mathrm{r}$ & $\mathrm{r}$ & $\mathrm{q}$ & 0 & $\mathrm{~s}$ \\
\hline $\mathrm{s}$ & $\mathrm{s}$ & $\mathrm{s}$ & $\mathrm{r}$ & $\mathrm{q}$ & 0 \\
\hline
\end{tabular}

Subsequently, $(\Omega ; \odot, 0)$ is a BCI-algebra.

Let $\Delta=\{\theta, \vartheta, \kappa\}$ be a set of parameters and consider the IFSS $(\mathrm{Y}, \Delta)$ over $\Omega$. Then $\mathrm{Y}[\theta], \mathrm{Y}[\vartheta]$, and $\mathrm{Y}[\kappa]$ are IFSs over $\Omega$ represented by:

\begin{tabular}{|c|c|c|c|c|c|}
\hline $\mathrm{Y}$ & 0 & $\mathrm{p}$ & $\mathrm{q}$ & $\mathrm{r}$ & $\mathrm{s}$ \\
\hline$\theta$ & {$[0.1,0.9]$} & {$[0.4,0.4]$} & {$[0.3,0.6]$} & {$[0.2,0.8]$} & {$[0.5,0.1]$} \\
\hline$\vartheta$ & {$[0,0.9]$} & {$[0.1,0.7]$} & {$[0.4,0.4]$} & {$[0.3,0.5]$} & {$[0.2,0.6]$} \\
\hline$\kappa$ & {$[0,1]$} & {$[0.2,0.6]$} & {$[0.3,0.5]$} & {$[0.4,0.3]$} & {$[0.1,0.7]$} \\
\hline
\end{tabular}

Afterwards, $(\mathrm{Y}, \Delta)$ is an AIFSID of $\Omega$, but since

$\xi_{Y[\vartheta]}(p \odot s)=\xi_{Y[\vartheta]}(q)=0.4 \not \leq 0.2=\xi_{Y[\vartheta]}((s \odot 0) \odot(0 \odot p)) \vee \xi_{Y[\vartheta]}(0)$

and

$\zeta_{Y[\vartheta]}(p \odot s)=\zeta_{Y[\vartheta]}(q)=0.4 \nsupseteq 0.6=\zeta_{Y[\vartheta]}((s \odot 0) \odot(0 \odot p)) \wedge \zeta_{Y[\theta]}(0)$,

i.e., $\mathrm{Y}[\vartheta]=\left\{\left(\xi_{\mathrm{Y}[\vartheta]}(l), \zeta_{\mathrm{Y}[\vartheta]}(l)\right): l \in \Omega\right.$ and $\left.\vartheta \in \Delta\right\}$ is not an AIFAID of $\Omega$.

Therefore $(\mathrm{Y}, \Delta)$ is not an AIFSAID of $\Omega$ with respect to $\vartheta$.

Hence $(\mathrm{Y}, \Delta)$ is not an AIFSAID of $\Omega$.

Theorem 2. Let $(\mathrm{Y}, \Delta)$ be an AIFSID over $\Omega$. If for any $\omega \in \Delta$ and $l, m \in \Omega, \xi_{Y}[\omega](m \odot l) \leq \xi_{Y}[\omega](l \odot$ $(0 \odot m))$ and $\zeta_{\mathrm{Y}[\omega]}(m \odot l) \geq \zeta_{\mathrm{Y}[\omega]}(l \odot(0 \odot m))$, then $(\mathrm{Y}, \Delta)$ is an AIFSAID over $\Omega$.

Proof. Let $(\mathrm{Y}, \Delta)$ be an AIFSID over $\Omega$.

Therefore, $\mathrm{Y}[\omega]=\left\{\left(\xi_{\mathrm{Y}[\omega]}(l), \zeta_{\mathrm{Y}[\omega]}(l)\right): l \in \Omega\right.$ and $\left.\omega \in \Delta\right\}$ is an AIFID of $\Omega$.

Thus, for any $\omega \in \Delta$ and $l, m, n \in \Omega$,

$\xi_{Y[\omega]}(m \odot l) \leq \xi_{Y[\omega]}(l \odot(0 \odot m))$

$$
\leq \tilde{\zeta}_{Y[\omega]}((l \odot(0 \odot m)) \odot n) \vee \xi_{Y[\omega]}(n)
$$

$\xi_{Y[\omega]}(m \odot l) \leq \xi_{Y[\omega]}((l \odot n) \odot(0 \odot m)) \vee \xi_{Y[\omega]}(n)$

and

$\zeta_{Y[\omega]}(m \odot l) \geq \zeta_{Y[\omega]}(l \odot(0 \odot m))$

$$
\geq \zeta_{Y[\omega]}((l \odot(0 \odot m)) \odot n) \wedge \zeta_{Y[\omega]}(n)
$$

$\zeta_{Y[\omega]}(m \odot l) \geq \zeta_{Y[\omega]}((l \odot n) \odot(0 \odot m)) \wedge \zeta_{Y[\omega]}(n)$

$\mathrm{Y}[\omega]=\left\{\left(\xi_{\mathrm{Y}[\omega]}(l), \zeta_{\mathrm{Y}[\omega]}(l)\right): l \in \Omega\right.$ and $\left.\omega \in \Delta\right\}$ is an AIFAID of $\Omega$.

Hence $(Y, \Delta)$ is an AIFSAID over $\Omega$.

Theorem 3. If $(\mathrm{Y}, \Delta)$ is an AIFSAID of $\Omega$, then for any parameter $\omega \in \Delta$ and $l, m, n \in \Omega, \xi_{\mathrm{Y}[\omega]}((l \odot n) \odot$ $(0 \odot m)) \leq \xi_{Y[\omega]}(l \odot(n \odot m))$ and $\zeta_{Y[\omega]}((l \odot n) \odot(0 \odot m)) \geq \zeta_{Y[\omega]}(l \odot(n \odot m))$.

Proof. Let $(\mathrm{Y}, \Delta)$ be an AIFSAID of $\Omega$.

Because $(l \odot n) \odot(0 \odot m)=(l \odot n) \odot((n \odot m) \odot n) \leq l \odot(n \odot m)$.

Therefore, $(l \odot n) \odot(0 \odot m) \odot(l \odot(n \odot m))=0$.

By Theorem $1,(\mathrm{Y}, \Delta)$ is an AIFSID of $\Omega$.

Thus, $\mathrm{Y}[\omega]=\left\{\left(\xi_{\mathrm{Y}[\omega]}(l), \zeta_{\mathrm{Y}[\omega]}(l)\right): l \in \Omega\right.$ and $\left.\omega \in \Delta\right\}$ is an AIFID of $\Omega$.

Thus, for every $l, m, n \in \Omega$ and $\omega \in \Delta$,

$\xi_{Y[\omega]}((l \odot n) \odot(0 \odot m)) \leq \xi_{Y[\omega]}(((l \odot n) \odot(0 \odot m)) \odot(l \odot(n \odot m))) \vee \xi_{Y[\omega]}(l \odot(n \odot m))$

$=\xi_{Y[\omega]}(0) \vee \xi_{Y[\omega]}(l \odot(n \odot m))$

$\leq \xi_{Y[\omega]}(l \odot(n \odot m))$ 
and

$$
\begin{aligned}
\zeta_{Y[\omega]}((l \odot n) \odot(0 \odot m)) & \geq \zeta_{Y[\omega]}(((l \odot n) \odot(0 \odot m)) \odot(l \odot(n \odot m))) \wedge \zeta_{Y[\omega]}(l \odot(n \odot m)) \\
& =\zeta_{Y[\omega]}(0) \wedge \zeta_{Y[\omega]}(l \odot(n \odot m)) \\
& \geq \zeta_{Y[\omega]}(l \odot(n \odot m)) . \square
\end{aligned}
$$

Definition 8. Let $(\mathrm{Y}, \Delta)$ and $(\Gamma, \Psi)$ be two IFSSs over $\Omega$. Then $(\mathrm{Y}, \Delta)$ "AND" $(\Gamma, \Psi)$ written as $(\mathrm{Y}, \Delta) \widetilde{\wedge}(\Gamma, \Psi)$ is $(\Pi, \Delta \times \Psi)$ of $\Omega$, where $\Pi[\omega, \omega]=\mathrm{Y}[\omega] \cap \Gamma[\omega]$ for all $(\omega, \omega) \in \Delta \times \Psi$.

Theorem 4. If $(\mathrm{Y}, \Delta)$ and $(\Gamma, \Psi)$ are two AIFSAIDs of $\Omega$, then $(\Pi, \Delta \times \Psi)$ is also an AIFSAID of $\Omega$.

Proof. By definition, $(\mathrm{Y}, \Delta) \widetilde{\wedge}(\Gamma, \Psi)=(\Pi, \Delta \times \Psi)$, where

$\Pi[\omega, \omega]=\mathrm{Y}[\omega] \cap \Gamma[\omega]=\left\{\left(\xi_{\mathrm{Y}[\omega] \cap \Gamma[\omega]}(l), \zeta_{\mathrm{Y}[\omega] \cap \Gamma[\omega]}(l)\right): l \in \Omega\right.$ and $\left.(\omega, \omega) \in \Delta \times \Psi\right\}$

For any $l \in \Omega$ and $(\omega, \omega) \in \Delta \times \Psi$,

$$
\begin{aligned}
\xi_{\Pi[[\omega, \omega]}(0)= & \xi_{Y[\omega] \cap \Gamma[\omega]}(0) \\
& =\xi_{Y[\omega]}(0) \vee \xi_{\Gamma[\omega]}(0) \\
& \leq \xi_{Y[\omega]}(l) \vee \xi_{\Gamma[\omega]}(l) \\
& =\xi_{Y[\omega] \cap \Gamma[\omega]}(l) \\
\xi_{\Pi[\omega, \omega]}(0) \leq & \xi_{\Pi[\omega, \omega]}(l)
\end{aligned}
$$

and

$$
\begin{aligned}
& \zeta_{\Pi[\omega, \omega]}(0)= \zeta_{Y[\omega] \cap \Gamma[\omega]}(0) \\
&=\zeta_{Y[\omega]}(0) \wedge \zeta_{\Gamma[\omega]}(0) \\
& \geq \zeta_{Y[\omega]}(l) \wedge \zeta_{\Gamma[\omega]}(l) \\
&=\zeta_{Y[\omega] \cap \Gamma[\omega]}(l) \\
& \zeta_{\Pi[\omega, \omega]}(0) \geq \zeta_{\Pi[\omega, \omega]}(l) .
\end{aligned}
$$

For any $l, m, n \in \Omega$, and $(\omega, \omega) \in \Delta \times \Psi$,

$\xi_{\Pi[\omega, \omega]}(m \odot l)=\xi_{Y[\omega] \cap \Gamma[\omega]}(m \odot l)=\xi_{Y[\omega]}(m \odot l) \vee \xi_{\Gamma[\omega]}(m \odot l)$

$\leq\left(\xi_{Y[\omega]}((l \odot n) \odot(0 \odot m)) \vee \xi_{Y[\omega]}(n)\right) \vee\left(\xi_{\Gamma[\omega]}((l \odot n) \odot(0 \odot m)) \vee \xi_{\Gamma[\omega]}(n)\right)$

$=\left(\xi_{Y[\omega]}((l \odot n) \odot(0 \odot m)) \vee \xi_{\Gamma[\omega]}((l \odot n) \odot(0 \odot m))\right) \vee\left(\xi_{Y[\omega]}(n) \vee \xi_{\Gamma[\omega]}(n)\right)$

$=\left(\tilde{\xi}_{Y}[\omega] \cap \Gamma[\omega]((l \odot n) \odot(0 \odot m))\right) \vee\left(\xi_{Y}[\omega] \cap \Gamma[\omega](n)\right)$

and

$\zeta_{\Pi[\omega, \omega]}(m \odot l)=\zeta_{Y[\omega] \cap \Gamma[\omega]}(m \odot l)=\zeta_{Y[\omega]}(m \odot l) \wedge \varsigma_{\Gamma[\omega]}(m \odot l)$

$\geq\left(\zeta_{Y[\omega]}((l \odot n) \odot(0 \odot m)) \wedge \zeta_{Y[\omega]}(n)\right) \wedge\left(\zeta_{\Gamma[\omega]}((l \odot n) \odot(0 \odot m)) \wedge \zeta_{\Gamma[\omega]}(n)\right)$

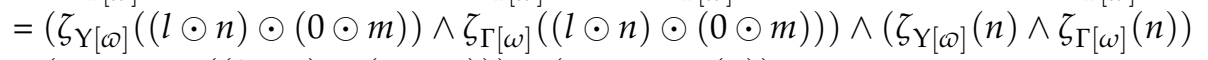

$=\left(\zeta_{Y[\omega] \cap \Gamma[\omega]}((l \odot n) \odot(0 \odot m))\right) \wedge\left(\zeta_{Y[\omega] \cap \Gamma[\omega]}(n)\right)$.

Thus, $\Pi[\omega, \omega]=\mathrm{Y}[\boldsymbol{\omega}] \cap \Gamma[\omega]$ is an AIFAID of $\Omega$ for any $(\omega, \omega) \in \Delta \times \Psi$.

Hence $(\Pi, \Delta \times \Psi)$ is an AIFSAID of $\Omega$ for any $(\omega, \omega) \in \Delta \times \Psi$.

Definition 9. The "extended intersection" of two IFSSs $(\mathrm{Y}, \Delta)$ and $(\Gamma, \Psi)$ denoted by $(\mathrm{Y}, \Delta) \sqcap_{E}(\Gamma, \Psi)$ is $(\Pi, \Theta)$, where $\Theta=\Delta \cup \Psi$ and for every $\omega \in \Theta$,

$$
\Pi(\omega)=\left\{\begin{array}{cl}
Y[\omega], & \omega \in \Delta-\Psi, \\
\Gamma[\omega], & \omega \in \Psi-\Delta, \\
Y[\omega] \cap \Gamma[\omega], & \omega \in \Delta \cap \Psi .
\end{array}\right.
$$

Theorem 5. If $(\mathrm{Y}, \Delta)$ and $(\Gamma, \Psi)$ are AIFSAIDs of $\Omega$, then $(\mathrm{Y}, \Delta) \sqcap_{E}(\Gamma, \Psi)$ is an AIFSAID of $\Omega$.

Proof. We know that $(Y, \Delta) \sqcap_{E}(\Gamma, \Psi)=(\Pi, \Theta)$, where $\Theta=\Delta \cup \Psi$ and for every $\omega \in \Theta$,

$$
\Pi(\omega)=\left\{\begin{array}{cc}
Y[\omega], & \omega \in \Delta-\Psi, \\
\Gamma[\omega], & \omega \in \Psi-\Delta, \\
Y[\mathcal{\omega}] \cap \Gamma[\omega], & \omega \in \Delta \cap \Psi .
\end{array}\right.
$$


For any $\omega \in \Theta$, if $\omega \in \Delta-\Psi$, then $\Pi(\omega)=Y(\omega)$ is an AIFAID of $\Omega$.

Likewise, if $\omega \in \Psi-\Delta, \Pi(\omega)=\Gamma(\omega)$, which is an AIFAID of $\Omega$.

Moreover if $\omega \in \Theta$, such that $\omega \in \Delta \cap \Psi$, then $\Pi(\omega)=\mathrm{Y}[\boldsymbol{\omega}] \cap \Gamma[\omega]$ is also an AIFAID of $\Omega$.

Therefore, $\Pi(\omega)$ is an AIFAID of $\Omega$.

Hence, $(\Pi, \Theta)$ is an AIFSAID of $\Omega$.

We deduce the following Corollary.

Corollary 1. The "restricted intersection" of two AIFSAIDs is an AIFSAID.

Definition 10. Let $(Y, \Delta)$ and $(\Gamma, \Psi)$ be two IFSSs over $\Omega$. Subsequently, the "union" denoted by $(\mathrm{Y}, \Delta) \widetilde{\cup}(\Gamma, \Psi)$ is $(\Pi, \Theta)$, where $\Theta=\Delta \cup \Psi$ and for every $\omega \in \Theta$,

$$
\Pi(\omega)=\left\{\begin{array}{cc}
Y[\omega], & \omega \in \Delta-\Psi, \\
\Gamma[\omega], & \omega \in \Psi-\Delta, \\
Y[\omega] \cup \Gamma[\omega], & \omega \in \Delta \cap \Psi .
\end{array}\right.
$$

The union of two AIFSAIDs is not necessarily an AIFSAID, as shown in the next example.

Example 3. Let $\Omega=\{0, p, q, r, s\}$ with Cayley table given by:

\begin{tabular}{|c|c|c|c|c|c|}
\hline$\odot$ & 0 & $\mathrm{p}$ & $\mathrm{q}$ & $\mathrm{r}$ & $\mathrm{s}$ \\
\hline 0 & 0 & 0 & $\mathrm{q}$ & $\mathrm{r}$ & $\mathrm{s}$ \\
\hline $\mathrm{p}$ & $\mathrm{p}$ & 0 & $\mathrm{q}$ & $\mathrm{r}$ & $\mathrm{s}$ \\
\hline $\mathrm{q}$ & $\mathrm{q}$ & $\mathrm{q}$ & 0 & $\mathrm{~s}$ & $\mathrm{r}$ \\
\hline $\mathrm{r}$ & $\mathrm{r}$ & $\mathrm{r}$ & $\mathrm{s}$ & 0 & $\mathrm{q}$ \\
\hline $\mathrm{s}$ & $\mathrm{s}$ & $\mathrm{s}$ & $\mathrm{r}$ & $\mathrm{q}$ & 0 \\
\hline
\end{tabular}

Subsequently, $(\Omega ; \odot, 0)$ is a $B C I$-algebra.

Let $\Delta=\{\theta, \vartheta, \kappa, \delta\}$ and $\Psi=\{\kappa, \delta, \eta\}$ be two collections of parameters and consider the IFSS $(\mathrm{Y}, \Delta)$ over $\Omega$. Afterwards, $\mathrm{Y}[\theta], \mathrm{Y}[\vartheta], \mathrm{Y}[\kappa]$ and $\mathrm{Y}[\delta]$ are IFSs over $\Omega$ given by:

\begin{tabular}{|c|c|c|c|c|c|}
\hline $\mathrm{Y}$ & 0 & $\mathrm{p}$ & $\mathrm{q}$ & $\mathrm{r}$ & $\mathrm{s}$ \\
\hline$\theta$ & {$[0,0.9]$} & {$[0,0.9]$} & {$[0.3,0.4]$} & {$[0.1,0.4]$} & {$[0.3,0.4]$} \\
\hline$\vartheta$ & {$[0.2,0.6]$} & {$[0.2,0.6]$} & {$[0.4,0.3]$} & {$[0.4,0.3]$} & {$[0.3,0.5]$} \\
\hline$\kappa$ & {$[0.1,0.8]$} & {$[0.1,0.8]$} & {$[0.5,0.2]$} & {$[0.3,0.5]$} & {$[0.5,0.2]$} \\
\hline$\delta$ & {$[0.2,0.7]$} & {$[0.2,0.7]$} & {$[0.3,0.5]$} & {$[0.5,0.3]$} & {$[0.5,0.3]$} \\
\hline
\end{tabular}

Then $\mathrm{Y}[\boldsymbol{\omega}]$ is an AIFAID of $\Omega$ with respect to $\theta, \vartheta, \kappa$, and $\delta$.

Thus $(\mathrm{Y}, \Delta)$ is an AIFSAID of $\Omega$.

Now let $(\Gamma, \Psi)$ be an IFSS over $\Omega$. Then $\Gamma[\kappa], \Gamma[\delta]$ and $\Gamma[\eta]$ are IFSs over $\Omega$ given by:

\begin{tabular}{|c|c|c|c|c|c|}
\hline$\Gamma$ & 0 & $\mathrm{p}$ & $\mathrm{q}$ & $\mathrm{r}$ & $\mathrm{s}$ \\
\hline $\mathcal{\kappa}$ & {$[0,0.7]$} & {$[0,0.7]$} & {$[0.3,0.5]$} & {$[0.5,0.2]$} & {$[0.5,0.2]$} \\
\hline$\delta$ & {$[0.2,0.6]$} & {$[0.2,0.6]$} & {$[0.5,0.2]$} & {$[0.5,0.2]$} & {$[0.3,0.4]$} \\
\hline$\eta$ & {$[0,0.9]$} & {$[0,0.9]$} & {$[0.3,0.4]$} & {$[0.1,0.6]$} & {$[0.3,0.4]$} \\
\hline
\end{tabular}

Subsequently, $\Gamma[\omega]$ is an AIFAID of $\Omega$ with respect to $\kappa, \delta$, and $\eta$.

Thus, $(\Gamma, \Psi)$ is an AIFSAID of $\Omega$.

Note that $(\mathrm{Y}, \Delta) \widetilde{\cup}(\Gamma, \Psi)=(\Pi, \Theta)$ is not an AIFSAID of $\Omega$ based on $\kappa \in \Delta \cap \Psi$. If $\Delta \cap \Psi=\varnothing$, then the union is an AIFSAID of $\Omega$ proved in the next theorem.

Theorem 6. Let $(\mathrm{Y}, \Delta)$ and $(\Gamma, \Psi)$ be two AIFSAIDs of $\Omega$. If $\Delta \cap \Psi=\varnothing$, then $(\mathrm{Y}, \Delta) \widetilde{\cup}(\Gamma, \Psi)=(\Pi, \Theta)$ is an AIFSAID of $\Omega$. 
Proof. We know that $(Y, \Delta) \widetilde{\cup}(\Gamma, \Psi)=(\Pi, \Theta)$, where $\Theta=\Delta \cup \Psi$ and for every $\omega \in \Theta$,

$$
\Pi(\omega)=\left\{\begin{array}{cc}
Y[\omega], & \omega \in \Delta-\Psi, \\
\Gamma[\omega], & \omega \in \Psi-\Delta, \\
Y[\omega] \cup \Gamma[\omega], & \omega \in \Delta \cap \Psi .
\end{array}\right.
$$

Because $\Delta \cap \Psi=\varnothing$, then either $\omega \in \Delta-\Psi$ or $\omega \in \Psi-\Delta$ for all $\omega \in \Theta$.

If $\omega \in \Delta-\Psi$, then $\Pi(\omega)=\mathrm{Y}(\omega)$, which is an AIFAID of $\Omega$.

Thus, $(\mathrm{Y}, \Delta)$ is an AIFSAID of $\Omega$.

Similarly $\mathscr{\omega} \in \Psi-\Delta$, then $\Pi(\mathscr{\omega})=\Gamma(\mathcal{\omega})$ is an AIFAID of $\Omega$.

Thus, $(\Gamma, \Psi)$ is an AIFSAID of $\Omega$.

Hence, $(Y, \Delta) \widetilde{\cup}(\Gamma, \Psi)$ is an AIFSAID of $\Omega$.

Definition 11. Let $(\mathrm{Y}, \Delta)$ be an anti-soft BCI-algebra (abbr. $\left.A S_{B C I} A\right)$ over $\Omega$. An IFSS $(\Gamma, \Psi)$ over $\Omega$ is an $\operatorname{AIFSID~of~}(\mathrm{Y}, \Delta)$, written as $(\Gamma, \Psi) \widetilde{\mathbf{\Lambda}}(\mathrm{Y}, \Delta)$, if $\Psi \subset \Delta$ and for any $\omega \in \Psi$,

$$
\Gamma[\omega]=\left\{\left(\xi_{\Gamma[\omega]}(l), \zeta_{\Gamma[\omega]}(l)\right): l \in \Omega\right\} \mathbf{\Lambda}[\omega] .
$$

Definition 12. Let $(Y, \Delta)$ be an $A S_{B C I}$ A over $\Omega$. An IFSS $(\Gamma, \Psi)$ over $\Omega$ is an AIFSAID of $(Y, \Delta)$, denoted by $(\Gamma, \Psi) \widetilde{\mathbf{\Lambda}}_{a}(\mathrm{Y}, \Delta)$, if $\Psi \subset \Delta$ and for any $\omega \in \Psi$,

$$
\Gamma[\omega]=\left\{\left(\xi_{\Gamma[\omega]}(l), \zeta_{\Gamma[\omega]}(l)\right): l \in \Omega\right\} \boldsymbol{\Delta}_{a} \mathrm{Y}[\omega] .
$$

Example 4. Let $\Omega=\{0, p, q, r, s\}$ with Cayley table:

\begin{tabular}{|c|c|c|c|c|c|}
\hline$\odot$ & 0 & $\mathrm{p}$ & $\mathrm{q}$ & $\mathrm{r}$ & $\mathrm{s}$ \\
\hline 0 & 0 & 0 & $\mathrm{q}$ & $\mathrm{r}$ & $\mathrm{s}$ \\
\hline $\mathrm{p}$ & $\mathrm{p}$ & 0 & $\mathrm{q}$ & $\mathrm{r}$ & $\mathrm{s}$ \\
\hline $\mathrm{q}$ & $\mathrm{q}$ & $\mathrm{q}$ & 0 & $\mathrm{~s}$ & $\mathrm{r}$ \\
\hline $\mathrm{r}$ & $\mathrm{r}$ & $\mathrm{r}$ & $\mathrm{s}$ & 0 & $\mathrm{q}$ \\
\hline $\mathrm{s}$ & $\mathrm{s}$ & $\mathrm{s}$ & $\mathrm{r}$ & $\mathrm{q}$ & 0 \\
\hline
\end{tabular}

Subsequently, $(\Omega ; \odot, 0)$ is a BCI-algebra.

Let $\Delta=\{\theta, \vartheta, \kappa\}$ be a set of parameters and let $(\mathrm{Y}, \Delta)$ be a soft set over $\Omega$ and so let $\mathrm{Y}[\theta]=\mathrm{Y}[\vartheta]=\{0, q, r, s\}$, $\mathrm{Y}[\kappa]=\{0, q\}$, that are all sub-algebras of $\Omega$.

Hence, $(\mathrm{Y}, \Delta)$ is an $A S_{B C I} A$ over $\Omega$.

Let $(\Gamma, \Psi)$ be an IFSS over $\Omega$, where $\Psi=\{\theta, \vartheta\} \subset \Delta$. Afterwards, $\Gamma[\theta]$ and $\Gamma[\vartheta]$ are IFSs in $\Omega$ defined by:

\begin{tabular}{|c|c|c|c|c|c|}
\hline$\Gamma$ & 0 & $\mathrm{p}$ & $\mathrm{q}$ & $\mathrm{r}$ & $\mathrm{s}$ \\
\hline$\theta$ & {$[0.2,0.7]$} & {$[0.2,0.7]$} & {$[0.2,0.7]$} & {$[0.4,0.1]$} & {$[0.4,0.1]$} \\
\hline$\vartheta$ & {$[0.3,0.7]$} & {$[0.3,0.7]$} & {$[0.3,0.7]$} & {$[0.5,0.4]$} & {$[0.5,0.4]$} \\
\hline
\end{tabular}

Afterwards, $\Gamma[\theta]=\left\{\left(\xi_{\Gamma[\theta]}(l), \zeta_{\Gamma[\theta]}(l)\right): l \in \Omega\right\}$ and $\Gamma[\vartheta]=\left\{\left(\xi_{\Gamma[\vartheta]}(l), \zeta_{\Gamma[\vartheta]}(l)\right): l \in \Omega\right\}$ are AIFAIDs of $\Omega$ related to $\Gamma[\theta]$ and $\Gamma[\vartheta]$, respectively.

Hence, $(\Gamma, \Psi) \widetilde{\mathbf{\Delta}}_{a}(\mathrm{Y}, \Delta)$.

Any $\operatorname{AIFSAID}(\Gamma, \Psi)$ of an $A S_{B C I} A(\mathrm{Y}, \Delta)$ is an $\operatorname{AIFSID}$ of $(\mathrm{Y}, \Delta)$, but the converse is not true, as proved by the next example.

Example 5. Let $\Omega=\{0, p, q, r, s\}$ with Cayley table. 


\begin{tabular}{|c|c|c|c|c|c|}
\hline$\odot$ & 0 & $\mathrm{p}$ & $\mathrm{q}$ & $\mathrm{r}$ & $\mathrm{s}$ \\
\hline 0 & 0 & 0 & 0 & 0 & 0 \\
\hline $\mathrm{p}$ & $\mathrm{p}$ & 0 & 0 & 0 & 0 \\
\hline $\mathrm{q}$ & $\mathrm{q}$ & $\mathrm{q}$ & 0 & $\mathrm{q}$ & 0 \\
\hline $\mathrm{r}$ & $\mathrm{r}$ & $\mathrm{r}$ & $\mathrm{r}$ & 0 & 0 \\
\hline $\mathrm{s}$ & $\mathrm{s}$ & $\mathrm{s}$ & $\mathrm{r}$ & $\mathrm{q}$ & 0 \\
\hline
\end{tabular}

Subsequently, $(\Omega ; \odot, 0)$ is a "BCK-algebra" and, thus, a "BCI-algebra".

Let $\Delta=\{\theta, \vartheta, \kappa, \delta, \eta\}$ be a set of parameters.

Let $(\mathrm{Y}, \Delta)$ be a soft set over $\Omega$ and so we let $\mathrm{Y}[\theta]=\Omega, \mathrm{Y}[\vartheta]=\mathrm{Y}[\kappa]=\{0, q, r, s\}$ and $\mathrm{Y}[\delta]=\mathrm{Y}[\eta]=\{0, q\}$, that are all subalgebras of $\Omega$.

Hence, $(\mathrm{Y}, \Delta)$ is a $A S_{B C I} A$ over $\Omega$.

Suppose that $(\Gamma, \Psi)$ is an IFSS over $\Omega$, where $\Psi=\{\kappa, \delta, \eta\} \subset \Delta$. Afterwards, $\Gamma[\kappa], \Gamma[\delta]$ and $\Gamma[\eta]$ are an IFSs in $\Omega$ represented by:

\begin{tabular}{|c|c|c|c|c|c|}
\hline$\Gamma$ & 0 & $\mathrm{p}$ & $\mathrm{q}$ & $\mathrm{r}$ & $\mathrm{s}$ \\
\hline$\kappa$ & {$[0,0.7]$} & {$[0.1,0.6]$} & {$[0.2,0.5]$} & {$[0.3,0.3]$} & {$[0.3,0.3]$} \\
\hline$\delta$ & {$[0.1,0.8]$} & {$[0.2,0.7]$} & {$[0.3,0.6]$} & {$[0.4,0.4]$} & {$[0.4,0.4]$} \\
\hline$\eta$ & {$[0.1,0.5]$} & {$[0.2,0.4]$} & {$[0.3,0.3]$} & {$[0.4,0.1]$} & {$[0.4,0.1]$} \\
\hline
\end{tabular}

Subsequently, $(\Gamma, \Psi)$ is an AIFSID of $(\mathrm{Y}, \Delta)$, but since

$\xi_{Y[k]}(r \odot q)=\xi_{Y[k]}(r)=0.3 \not \leq 0.2=\xi_{Y[k]}((q \odot 0) \odot(0 \odot r)) \vee \xi_{Y[\omega]}(0)$

and

$\zeta_{Y[\kappa]}(r \odot q)=\zeta_{Y[\kappa]}(r)=0.3 \nsupseteq 0.5=\xi_{Y[k]}((q \odot 0) \odot(0 \odot r)) \wedge \zeta_{Y[\omega]}(0)$.

i.e., $\Gamma[\kappa]=\left\{\left(\xi_{\Gamma[\kappa]}(l), \zeta_{\Gamma[\kappa]}(l)\right): l \in \Omega\right\}$ is not an AIFAID of $\Omega$ related to $\mathrm{Y}[\kappa]$.

Therefore $(\Gamma, \Psi)$ is not an AIFSAID of $A S_{B C I} A(Y, \Delta)$.

Theorem 7. Let $(Y, \Delta)$ be an $A S_{B C I} A$ over $\Omega$. If $(\Gamma, \Psi)$ and $(\Pi, \Lambda)$ are AIFSAIDs of $(Y, \Delta)$, then the "extended intersection" of $(\Gamma, \Psi)$ and $(\Pi, \Lambda)$ is an AIFSAIDs of $(\mathrm{Y}, \Delta)$.

Proof. We know that $(\Gamma, \Psi) \sqcap_{E}(\Pi, \Lambda)=(\Xi, \Theta)$, where $\Theta=\Psi \cup \Lambda \subset \Delta$ and for every $\omega \in \Theta$,

$$
\Xi(\omega)=\left\{\begin{array}{cc}
\Gamma[\omega], & \omega \in \Psi-\Lambda, \\
\Pi[\omega], & \omega \in \Lambda-\Psi, \\
\Gamma[\omega] \cap \Pi[\omega], & \omega \in \Psi \cap \Lambda .
\end{array}\right.
$$

For any $\boldsymbol{\omega} \in \Theta$, if $\omega \in \Psi-\Lambda$, then $\Xi[\omega]=\Gamma[\omega]=\left\{\left(\xi_{\Gamma[\omega]}(l), \zeta_{\Gamma[\omega]}(l)\right): l \in \Omega\right\} \mathbf{\Delta}_{a} Y[\omega]$, since $(\Gamma, \Psi) \widetilde{\mathbf{\Delta}}_{a}(\mathrm{Y}, \Delta)$.

Likewise, if $\omega \in \Lambda-\Psi$, then $\Xi[\omega]=\Pi[\omega]=\left\{\left(\xi_{\Pi[\omega]}(l), \zeta_{\Pi[\omega]}(l)\right): l \in \Omega\right\} \boldsymbol{\Delta}_{a} Y[\omega]$, since $(\Pi, \Lambda) \widetilde{\mathbf{\Lambda}}_{a}(\mathrm{Y}, \Delta)$.

Moreover if $\omega \in \Theta$, such that $\omega \in \Psi \cap \Lambda$, then $\Xi(\omega)=\Gamma[\omega] \cap \Pi[\omega]=\left\{\left(\xi_{\Gamma[\omega]}(l) \vee \xi_{\Pi[\omega]}(l)\right),\left(\zeta_{\Gamma[\omega]}(l) \wedge\right.\right.$ $\left.\left.\zeta_{\Pi[\omega]}(l)\right)\right\} \boldsymbol{\Delta}_{a} \mathrm{Y}[\omega]$.

Therefore, $\Xi(\omega) \boldsymbol{\Lambda}_{a} Y[\omega]$ for any $\omega \in \Theta$.

Hence, $(\Xi, \Theta)=(\Gamma, \Psi) \sqcap_{E}(\Pi, \Lambda) \widetilde{\mathbf{\Lambda}}_{a}(\mathrm{Y}, \Delta)$.

Next corollary follows directly.

Corollary 2. Let $(\Gamma, \Psi)$ and $(\Pi, \Lambda)$ be two AIFSAIDs of an $A S_{B C I} A(Y, \Delta)$. If $\Psi \cap \Lambda=\varnothing$, then the "union" $(\Gamma, \Psi) \widetilde{\cup}(\Pi, \Lambda)$ is an AIFSAID of $(\mathrm{Y}, \Delta)$. 


\section{Characterization of Anti-Intuitionistic Fuzzy Soft a-Ideals}

In this section, we give characterizations of an $\operatorname{AIFSAID}(\mathrm{Y}, \Delta)$ over $\Omega$ while using the idea of a soft $(\gamma, v)$-level set, $L(\mathrm{Y}[\omega] ; \gamma ; v)=\left\{l \in \Omega \mid \xi_{Y[\omega]}(l) \leq \gamma\right.$ and $\left.\zeta_{Y[\omega]}(l) \geq v\right\}$, for any $\omega \in \Delta$ and $\gamma, v \in[0,1]$.

Theorem 8. An AIFSS $(\mathrm{Y}, \Delta)$ over $\Omega$ is an AIFSAID over $\Omega \Longleftrightarrow$ the non-empty soft $(\gamma, v)$-level set, $L(\mathrm{Y}[\omega] ; \gamma ; v)=\left\{l \in \Omega \mid \xi_{\mathrm{Y}[\omega]}(l) \leq \gamma\right.$ and $\left.\zeta_{\mathrm{Y}[\omega]}(l) \geq v\right\}$ is an a-ideal of $\Omega$, for any $\omega \in \Delta$ and $\gamma, v \in[0,1]$.

Proof. Let $(\mathrm{Y}, \Delta)$ be an AIFSAID over $\Omega$.

Afterwards, $\mathrm{Y}[\omega]=\left\{\left(\xi_{\mathrm{Y}[\omega]}(l), \zeta_{\mathrm{Y}[\omega]}(l)\right) \mid l \in \Omega\right\}$ is an AIFAID of $\Omega$, for any $\omega \in \Delta$.

Let $L(\mathrm{Y}[\omega] ; \gamma ; v)=\left\{l \in \Omega \mid \xi_{\mathrm{Y}[\omega]}(l) \leq \gamma\right.$ and $\left.\zeta_{\mathrm{Y}[\omega]}(l) \geq v\right\} \neq \varnothing$, for any $\omega \in \Delta$ and $\gamma, v \in[0,1]$. Subsequently, for any $l \in L(\mathrm{Y}[\omega] ; \gamma ; v)$,

$\xi_{Y[\omega]}(0) \leq \xi_{Y[\omega]}(l) \leq \gamma$ and $\zeta_{Y[\omega]}(0) \geq \zeta_{Y[\omega]}(l) \geq v$,

i.e., $0 \in L(\mathrm{Y}[\omega] ; \gamma ; v)$.

Now, let $(l \odot n) \odot(0 \odot m) \in L(\mathrm{Y}[\omega] ; \gamma ; v)$ and $n \in L(\mathrm{Y}[\omega] ; \gamma ; v)$, for any $l, m, n \in \Omega$.

Subsequently, $\xi_{Y[\omega]}((l \odot n) \odot(0 \odot m)) \leq \gamma, \xi_{Y}[\omega](n) \leq \gamma$

and

$\zeta_{Y[\omega]}((l \odot n) \odot(0 \odot m)) \geq v, \zeta_{Y[\omega]}(n) \geq v$.

Thus, for any $l, m, n \in \Omega$,

$\xi_{Y[\omega]}(m \odot l) \leq \xi_{Y[\omega]}((l \odot n) \odot(0 \odot m)) \vee \xi_{Y[\omega]}(n) \leq \gamma$.

$\zeta_{Y[\omega]}(m \odot l) \geq \zeta_{Y[\omega]}((l \odot n) \odot(0 \odot m)) \wedge \zeta_{Y[\omega]}(n) \geq v$.

i.e., $m \odot l \in L(\mathrm{Y}[\omega] ; \gamma ; v)$.

Hence, $L(\mathrm{Y}[\omega] ; \gamma ; v) \neq \varnothing$ is an a-ideal of $\Omega$, for any $\omega \in \Delta$ and $\gamma, v \in[0,1]$.

Conversely assume that $L(\mathrm{Y}[\omega] ; \gamma ; v)$ is an a-ideal of $\Omega$, for any $\omega \in \Delta$ and $\gamma, v \in[0,1]$.

If for some $l_{0} \in \Omega$ and $\omega_{0} \in \Delta, \xi_{Y}\left[\omega_{0}\right](0)>\xi_{Y\left[\omega_{0}\right]}\left(l_{0}\right)$ and $\zeta_{Y\left[\omega_{0}\right]}(0)<\zeta_{Y\left[\omega_{0}\right]}\left(l_{0}\right)$, then $\xi_{Y\left[\omega_{0}\right]}(0)>\gamma_{0} \geq$ $\xi_{Y\left[\omega_{0}\right]}\left(l_{0}\right)$ and $\zeta_{Y\left[\omega_{0}\right]}(0)<v_{0} \leq \zeta_{Y\left[\omega_{0}\right]}\left(l_{0}\right)$, for some $\gamma_{0}, v_{0} \in[0,1]$.

This implies that $l_{0} \in L\left(\mathrm{Y}\left[\omega_{0}\right] ; \gamma_{0} ; v_{0}\right)$ and that $0 \notin L\left(\mathrm{Y}\left[\omega_{0}\right] ; \gamma_{0} ; v_{0}\right)$, this contradicts the hypothesis that $L\left(\mathrm{Y}\left[\omega_{0}\right] ; \gamma_{0} ; v_{0}\right)$ is an a-ideal of $\Omega$.

Thus $\xi_{Y[\omega]}(0) \leq \xi_{Y[\omega]}(l)$ and $\zeta_{Y[\omega]}(0) \geq \zeta_{Y[\omega]}(l)$, for any $\omega \in \Delta$ and $l \in \Omega$.

Moreover, if there are elements $l_{0}, m_{0}, n_{0} \in \Omega$ and $\omega_{0} \in \Delta$, such that

$\xi_{Y\left[\omega_{0}\right]}\left(m_{0} \odot l_{0}\right)>\xi_{Y\left[\omega_{0}\right]}\left(\left(l_{0} \odot n_{0}\right) \odot\left(0 \odot m_{0}\right)\right) \vee \xi_{Y\left[\omega_{0}\right]}\left(n_{0}\right)$

and

$\zeta_{\mathrm{Y}\left[\omega_{0}\right]}\left(m_{0} \odot l_{0}\right)<\zeta_{\mathrm{Y}\left[\omega_{0}\right]}\left(\left(l_{0} \odot n_{0}\right) \odot\left(0 \odot m_{0}\right)\right) \wedge \zeta_{\mathrm{Y}[\omega]}\left(n_{0}\right)$.

Afterwards, for some $\gamma_{0}, v_{0} \in[0,1]$,

$\xi_{Y\left[\omega_{0}\right]}\left(m_{0} \odot l_{0}\right)>\gamma_{0} \geq \xi_{Y\left[\omega_{0}\right]}\left(\left(l_{0} \odot n_{0}\right) \odot\left(0 \odot m_{0}\right)\right) \vee \xi_{Y\left[\omega_{0}\right]}\left(n_{0}\right)$

and

$\zeta_{Y\left[\omega_{0}\right]}\left(m_{0} \odot l_{0}\right)<v_{0} \leq \zeta_{Y\left[\omega_{0}\right]}\left(\left(l_{0} \odot n_{0}\right) \odot\left(0 \odot m_{0}\right)\right) \wedge \zeta_{Y[\omega]}\left(n_{0}\right)$.

i.e., $m_{0} \odot l_{0} \notin L\left(\mathrm{Y}\left[\omega_{0}\right] ; \gamma_{0} ; v_{0}\right)$, again a contradiction.

Thus, for any $l, m, n \in \Omega$ and for any $\omega \in \Delta$,

$\xi_{Y[\omega]}(m \odot l) \leq \xi_{Y[\omega]}((l \odot n) \odot(0 \odot m)) \vee \xi_{Y[\omega]}(n)$

and

$\zeta_{Y[\omega]}(m \odot l) \geq \zeta_{Y[\omega]}((l \odot n) \odot(0 \odot m)) \wedge \zeta_{Y[\omega]}(n)$

i.e., $\mathrm{Y}[\omega]=\left\{\left(\xi_{\mathrm{Y}[\omega]}(l), \zeta_{\mathrm{Y}[\omega]}(l)\right) \mid l \in \Omega\right\}$ is an AIFAID of $\Omega$, for any $\omega \in \Delta$.

Hence, $(\mathrm{Y}, \Delta)$ is an AIFSAID over $\Omega$.

From the above Theorem we get the following corollary.

Corollary 3. An AIFSS $(\mathrm{Y}, \Delta)$ over $\Omega$ is an AIFSAID over $\Omega \Longleftrightarrow$ the non-empty soft $(\gamma, v)$-level set, $L(\mathrm{Y}[\omega] ; \gamma ; v)=\left\{l \in \Omega \mid \xi_{\mathrm{Y}[\omega]}(l) \leq \gamma\right.$ and $\left.\zeta_{\mathrm{Y}[\omega]}(l) \geq v\right\}$, is an a-ideal of $\Omega$, for any $\omega \in \Delta$ and $\gamma, v \in$ $(1 / 2,1]$. 
Theorem 9. A non-empty soft $(\gamma, v)$-level set, $L(\mathrm{Y}[\omega] ; \gamma ; v)=\left\{l \in \Omega \mid \xi_{\mathrm{Y}[\omega]}(l) \leq \gamma\right.$ and $\left.\zeta_{\mathrm{Y}[\omega]}(l) \geq v\right\}$, is an a-ideal of $\Omega$, for any $\omega \in \Delta$ and $\gamma, v \in(1 / 2,1] \Longleftrightarrow$ the following conditions hold:

(i) $\left(\xi_{Y[\omega]}(0) \vee 1 / 2\right) \leq \xi_{Y[\omega]}(l)$ and $\left(\zeta_{Y[\omega]}(0) \vee 1 / 2\right) \geq \zeta_{Y[\omega]}(l)$,

(ii) $\left(\xi_{Y[\omega]}(m \odot l) \vee 1 / 2\right) \leq \xi_{Y[\omega]}((l \odot n) \odot(0 \odot m)) \vee \xi_{Y[\omega]}(n)$,

(iii) $\left(\zeta_{Y[\omega]}(m \odot l) \vee 1 / 2\right) \geq \zeta_{Y[\omega]}((l \odot n) \odot(0 \odot m)) \wedge \zeta_{Y[\omega]}(n)$,

for any $\omega \in \Delta$ and $l, m, n \in \Omega$.

Proof. Let the non-empty soft $(\gamma, v)$-level set, $L(\mathrm{Y}[\omega] ; \gamma ; v)=\left\{l \in \Omega \mid \xi_{\mathrm{Y}[\omega]}(l) \leq \gamma\right.$ and $\left.\zeta_{\mathrm{Y}[\omega]}(l) \geq v\right\}$ be an a-ideal of $\Omega$, for any $\omega \in \Delta$ and $\gamma, v \in(1 / 2,1]$.

If for some $l_{0} \in \Omega$ and $\omega_{0} \in \Delta$,

$\left(\xi_{Y\left[\omega_{0}\right]}(0) \vee 1 / 2\right)>\xi_{Y\left[\omega_{0}\right]}\left(l_{0}\right)$ and $\left(\xi_{Y\left[\omega_{0}\right]}(0) \vee 1 / 2\right)<\xi_{Y\left[\omega_{0}\right]}\left(l_{0}\right)$.

Then there are $\gamma_{0}, v_{0} \in(1 / 2,1]$, such that

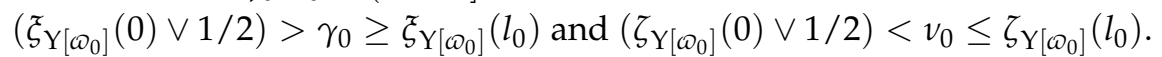

This implies that $\xi_{\mathrm{Y}\left[\omega_{0}\right]}(0)>\gamma_{0} \geq \xi_{\mathrm{Y}\left[\omega_{0}\right]}\left(l_{0}\right)$ and $\zeta_{\mathrm{Y}\left[\omega_{0}\right]}(0)<v_{0} \leq \zeta_{\mathrm{Y}\left[\omega_{0}\right]}\left(l_{0}\right)$.

i.e., $l_{0} \in L\left(\mathrm{Y}\left[\omega_{0}\right] ; \gamma_{0} ; v_{0}\right)$ but $0 \notin L\left(\mathrm{Y}\left[\omega_{0}\right] ; \gamma_{0} ; v_{0}\right)$, which gives a contradiction to the assumption that $L\left(\mathrm{Y}\left[\omega_{0}\right] ; \gamma_{0} ; v_{0}\right)$ is an a-ideal of $\Omega$, for any $\omega_{0} \in \Delta$ and $\gamma_{0}, v_{0} \in(1 / 2,1]$.

Thus, (i) is valid.

Moreover, if there are elements $l_{0}, m_{0}, n_{0} \in \Omega$ and $\omega_{0} \in \Delta$, such that

$\left(\xi_{Y\left[\omega_{0}\right]}\left(m_{0} \odot l_{0}\right) \vee 1 / 2\right)>\xi_{Y\left[\omega_{0}\right]}\left(\left(l_{0} \odot n_{0}\right) \odot\left(0 \odot m_{0}\right)\right) \vee \xi_{Y\left[\omega_{0}\right]}\left(n_{0}\right)$

and

$\left(\zeta_{\mathrm{Y}\left[\omega_{0}\right]}\left(m_{0} \odot l_{0}\right) \vee 1 / 2\right)<\zeta_{\mathrm{Y}\left[\omega_{0}\right]}\left(\left(l_{0} \odot n_{0}\right) \odot\left(0 \odot m_{0}\right)\right) \wedge \zeta_{\mathrm{Y}[\omega]}\left(n_{0}\right)$.

Subsequently, for some $\gamma_{0}, v_{0} \in(1 / 2,1]$,

$\left(\xi_{Y\left[\omega_{0}\right]}\left(m_{0} \odot l_{0}\right) \vee 1 / 2\right)>\gamma_{0} \geq \xi_{Y}\left[\omega_{0}\right]\left(\left(l_{0} \odot n_{0}\right) \odot\left(0 \odot m_{0}\right)\right) \vee \xi_{Y}\left[\omega_{0}\right]\left(n_{0}\right)$

and

$\left(\zeta_{Y\left[\omega_{0}\right]}\left(m_{0} \odot l_{0}\right) \vee 1 / 2\right)<v_{0} \leq \zeta_{Y\left[\omega_{0}\right]}\left(\left(l_{0} \odot n_{0}\right) \odot\left(0 \odot m_{0}\right)\right) \wedge \zeta_{Y[\omega]}\left(n_{0}\right)$.

i.e., $\xi_{Y}\left[\omega_{0}\right]\left(m_{0} \odot l_{0}\right)>\gamma_{0} \geq \xi_{Y}\left[\omega_{0}\right]\left(\left(l_{0} \odot n_{0}\right) \odot\left(0 \odot m_{0}\right)\right) \vee \xi_{Y}\left[\omega_{0}\right]$

and

$\zeta_{\mathrm{Y}\left[\omega_{0}\right]}\left(m_{0} \odot l_{0}\right)<v_{0} \leq \zeta_{\mathrm{Y}\left[\omega_{0}\right]}\left(\left(l_{0} \odot n_{0}\right) \odot\left(0 \odot m_{0}\right)\right) \wedge \zeta_{\mathrm{Y}[\omega]}\left(n_{0}\right)$.

i.e., $\left(\left(l_{0} \odot n_{0}\right) \odot\left(0 \odot m_{0}\right)\right) \in L\left(\mathrm{Y}\left[\omega_{0}\right] ; \gamma_{0} ; v_{0}\right)$ and $n_{0} \in L\left(\mathrm{Y}\left[\omega_{0}\right] ; \gamma_{0} ; v_{0}\right)$ but $\left(m_{0} \odot l_{0}\right) \notin L\left(\mathrm{Y}\left[\omega_{0}\right] ; \gamma_{0} ; v_{0}\right)$, which-again-contradicts the assumption that $L\left(\mathrm{Y}\left[\omega_{0}\right] ; \gamma_{0} ; v_{0}\right)$ is an a-ideal of $\Omega$, for any $\omega_{0} \in \Delta$ and $\gamma_{0}, v_{0} \in(1 / 2,1]$.

Hence, (ii) and (iii) are valid.

Conversely, suppose that the conditions (i), (ii), and (iii) are valid.

Let $L(\mathrm{Y}[\omega] ; \gamma ; v)=\left\{l \in \Omega \mid \xi_{\mathrm{Y}[\omega]}(l) \leq \gamma\right.$ and $\left.\zeta_{\mathrm{Y}[\omega]}(l) \geq v\right\} \neq \varnothing$, for any $\omega \in \Delta$ and $\gamma, v \in(1 / 2,1]$.

Subsequently, for any $l \in L(\mathrm{Y}[\omega] ; \gamma ; v)$,

$\left(\xi_{Y[\omega]}(0) \vee 1 / 2\right) \leq \xi_{Y[\omega]}(l) \leq \gamma$ and

$\left(\zeta_{Y[\omega]}(0) \vee 1 / 2\right) \geq \zeta_{Y[\omega]}(l) \geq v$

which implies $\xi_{Y[\omega]}(0) \leq \gamma$ and $\zeta_{Y[\omega]}(0) \geq v$.

Thus, $0 \in L(\mathrm{Y}[\omega] ; \gamma ; v)$.

Now let $(l \odot n) \odot(0 \odot m) \in L(\mathrm{Y}[\omega] ; \gamma ; v)$ and $n \in L(\mathrm{Y}[\omega] ; \gamma ; v)$, for any $l, m, n \in \Omega$.

Subsequently, $\tilde{\xi}_{Y[\omega]}((l \odot n) \odot(0 \odot m)) \leq \gamma, \xi_{Y[\omega]}(n) \leq \gamma$

and

$\zeta_{Y[\omega]}((l \odot n) \odot(0 \odot m)) \geq v, \zeta_{Y[\omega]}(n) \geq v$.

Thus, from (ii), we get,

$\left(\xi_{Y[\omega]}(m \odot l) \vee 1 / 2\right) \leq \xi_{Y[\omega]}((l \odot n) \odot(0 \odot m)) \vee \xi_{Y[\omega]}(n) \leq \gamma$

and

$\left(\zeta_{Y[\omega]}(m \odot l) \vee 1 / 2\right) \geq \zeta_{Y[\omega]}((l \odot n) \odot(0 \odot m)) \wedge \zeta_{Y[\omega]}(n) \geq v$.

This implies, $\xi_{Y[\omega]}(m \odot l) \leq \gamma$ and $\zeta_{Y[\omega]}(m \odot l) \geq v$.

Thus, $m \odot l \in L(\mathrm{Y}[\omega] ; \gamma ; v)$.

Therefore, $L(\mathrm{Y}[\omega] ; \gamma ; v)$ is an a-ideal of $\Omega$, for any $\omega \in \Delta$ and $\gamma, v \in(1 / 2,1]$. 


\section{Conclusions}

The notion of anti-intuitionistic fuzzy soft a-ideal (abbr. AIFSAID) is introduced and studied over a $B C I$-algebra $\Omega$. We proved that any AIFSAID is an anti-intuitionistic fuzzy soft ideal (abbr. AIFSID) of $\Omega$ and that the converse is not always true. We proved that the operations "AND", "extended intersection", and "restricted intersection" between any two AIFSAIDs of $\Omega$, is also an AIFSAID of $\Omega$ whereas the "union" is not necessarily an AIFSAID. Moreover, characterizations of AIFSAID using the concept of a soft level set were given.

Author Contributions: Conceptualization, G.M.; Formal analysis, G.M. and D.A.-K.; Investigation, D.A.-K. and M.B.; Methodology, G.M. and D.A.-K.; Writing_original draft, G.M.; Writing—review and editing, D.A.-K. and M.B. All authors contributed equally to this work. All the authors have read and approved the final version manuscript.

Funding: This research received no external funding.

Acknowledgments: The authors are grateful to the anonymous referees for a careful checking of the details and for helpful comments that improved the overall presentation of this paper.

Conflicts of Interest: The authors declare that they have no conflict of interest.

\section{References}

1. Zadeh, L.A. Fuzzy sets. Inform. Control 1965, 8, 338-353. [CrossRef]

2. Atanassov, K.T. Intuitionistic fuzzy sets. Fuzzy Sets Syst. 1986, 20, 87-96. [CrossRef]

3. Molodtsov, D. Soft set theory-first results. Global optimization, control, and games, III. Comput. Math. Appl. 1999, 37, 19-31. [CrossRef]

4. Maji, P.K.; Biswas, R.; Roy, A.R. Intuitionistic fuzzy soft sets. J. Fuzzy Math. 2001, 9, 677-692.

5. Iskei, K. An algebra related with a propositional calculus. Proc. Jpn. Acad. 1966, 42, 26-29. [CrossRef]

6. Jun, Y.B.; Kim, K.H. Intuitionistic fuzzy ideals of BCK-algebras. Int. J. Math. Math. Sci. 2000, 24, 839-849. [CrossRef]

7. Bej, T.; Pal, M. Doubt intuitionistic fuzzy $H$-ideals in BCK/BCI-algebras. Ann. Fuzzy Math. Inform. 2014, 8, 593-605.

8. Muhiuddin, G. Hesitant fuzzy filters and hesitant fuzzy G-filters in residuated lattices. J. Comput. Anal. Appl. 2016, 21, 394-404.

9. Muhiuddin, G.; Al-roqi, A.M. Unisoft Filters in $R_{0}$-algebras. J. Comput. Anal. Appl. 2015, 19, 133-143.

10. Muhiuddin, G.; Al-roqi, A.M. Cubic soft sets with applications in BCK/BCI-algebras. Ann. Fuzzy Math. Inform. 2014, 8, 291-304.

11. Muhiuddin, G.; Al-roqi, A.M.; Aldhafeeri, S. Filter theory in MTL-algebras based on Uni-soft property. Bull. Iran. Math. Soc. 2017, 43, 2293-2306.

12. Muhiuddin, G.; Aldhafeeri, S. Subalgebras and ideals in BCK/BCI-algebras based on uni-hesitant fuzzy set theory. Eur. J. Pure Appl. Math. 2018, 11, 417-430. [CrossRef]

13. Muhiuddin, G.; Aldhafeeri, S. N-Soft p-ideal of BCI-algebras. Eur. J. Pure Appl. Math. 2019, 12, $79-87$. [CrossRef]

14. Muhiuddin, G.; Feng, F.; Jun, Y.B. Subalgebras of BCK/BCI-algebras Based on Cubic Soft Sets. Sci. World J. 2014, 2014, 458638.10.1155/2014/458638. [CrossRef] [PubMed]

15. Muhiuddin, G.; Kim, H.S.; Song, S.Z.; Jun, Y.B. Hesitant fuzzy translations and extensions of subalgebras and ideals in BCK/BCI-algebras. J. Intelli. Fuzzy Syst. 2017, 32, 43-48. [CrossRef]

16. Muhiuddin, G.; Rehman, N.; Jun, Y.B. A generalization of $(\in, \in \vee q)$-fuzzy ideals in ternary semigroups. Ann. Commun. Math. 2019, 2, 73-83.

17. Muhiuddin, G.; Jun, Y.B. Sup-hesitant fuzzy subalgebras and its translations and extensions. Ann. Commun. Math. 2019, 2, 48-56.

18. Muhiuddin, G.; Balamurugan, M. Hesitant intuitionistic fuzzy soft b-ideals of BCK-algebras. Ann. Commun. Math. 2020, 3, 26-34.

19. Belov, A.Y.; Ivanov, I.A. Construction of Semigroups with Some Exotic Properties. Commun. Algebra 2003, 31, 673-696. [CrossRef] 
20. Al-roqi, A.M.; Muhiuddin, G.; Aldhafeeri, S. Normal Unisoft Filters in $R_{0}$-algebras. Cogent Math. 2017, 4, 1383006. [CrossRef]

21. Al-Kadi, D.; Muhiuddin, G. Bipolar fuzzy BCI-implicative ideals of BCI-algebras. Ann. Commun. Math. 2020, 3, 88-96.

22. Al-Masarwah, A.; Ahmad, A.G.; Muhiuddin, G. Doubt N-ideals theory in BCK-algebras based on N-structures. Ann. Commun. Math. 2020, 3, 54-62.

23. Al-Masarwah, A.; Ahmad, A.G. A new form of generalized m-PF Ideals in BCK/BCI-algebras. Ann. Commun. Math. 2019, 2, 11-16.

24. Thongarsa, S.; Burandate, P.; Iampan, A. Some operations of fuzzy sets in UP-algebras with respect to a triangular norm. Ann. Commun. Math. 2019, 2, 1-10.

25. Hong, S.M.; Jun, Y.B. Anti-fuzzy ideals in BCK-algebras. Kyungpook Math. J. 1998, 38, 145-150.

26. Ivanov-Pogodaeva, I.; Malev, S. Finite Gröbner basis algebras with unsolvable nilpotency problem and zero divisors problem. J. Algebra 2018, 508, 575-588. [CrossRef]

27. Senapati, T.; Shum, K.P. Cubic subalgebras of BCH-algebras. Ann. Commun. Math. 2018, 1, 65-73.

28. Jun, Y.B.; Ahn, S.S.; Muhiuddin, G. Hesitant fuzzy soft subalgebras and ideals in BCK/BCI-algebras. Sci. World J. 2014, 2014, 1383006. [CrossRef]

29. Jun, Y.B.; Song, S.Z.; Muhiuddin, G. Concave Soft Sets, Critical Soft Points, and Union-Soft Ideals of Ordered Semigroups. Sci. World J. 2014, 2014, 467968. [CrossRef]

30. Jun, Y.B.; Smarandache, F.; Bordbar, H. Neutrosophic $\mathcal{N}$-structures applied to $B C K / B C I$-algebras. Information 2017, 8, 128. [CrossRef]

31. Jun, Y.B.; Smarandache, F.; Song, S.Z.; Khan, M. Neutrosophic positive implicative $\mathcal{N}$-ideals in $B C K$-algebras. Axioms 2018, 7, 3. [CrossRef]

32. Li, Y.; Deng, Y. Intuitionistic evidence sets. IEEE Access 2019, 7, 106417-106426. [CrossRef]

33. Sun, C.; Li, S.; Deng, Y. Determining weights in multi-criteria decision making based on negation of probability distribution under uncertain environment. Mathematics 2020, 8, 191. [CrossRef]

34. Ejegwa, P.A.; Otuwe, J.A. Frattini fuzzy subgroups of fuzzy groups. Ann. Commun. Math. 2019, 2, $24-31$.

35. Xue, Y.; Deng, Y. Entailment for intuitionistic fuzzy sets based on generalized belief structures. Int. J. Intell. Syst. 2020, 35, 963-982. [CrossRef]

36. Balamurugan, M.; Balasubramanian, G.; Ragavan, C. Translations of intuitionistic fuzzy soft structure of B-algebras. Malaya J. Mat. 2018, 6, 685-700. [CrossRef]

(C) 2020 by the authors. Licensee MDPI, Basel, Switzerland. This article is an open access article distributed under the terms and conditions of the Creative Commons Attribution (CC BY) license (http:/ / creativecommons.org/licenses/by/4.0/). 